\title{
Lévy-Based Interest Rate Derivatives: Change of Time Method and PIDEs
}

\author{
AnATOLiY SWISHCHUK ${ }^{1}$ \\ University of Calgary
}

\begin{abstract}
In this paper, we show how to calculate the price of zerocoupon bonds for many Gaussian and Lévy one-factor and multi-factor models of $r(t)$ using change of time method. These models include, in particular, Ornshtein-Uhlenbeck (1930), Vasicek (1977), Cox-Ingersoll-Ross (1985), continuous-time GARCH, Ho-Lee (1986), Hull-White (1990) and HeathJarrow-Morton (1992) models and their various combinations. We also derive partial integro-differential equations (PIDEs) for the values of swaps, caps, floors and options on them, swaptions, captions and floortions, respectively. We apply the change of time method to price the interest rate derivatives for the interest rates $r(t)$ described by various stochastic differential equations driven by $\alpha$-stable Lévy processes.
\end{abstract}

\section{Introduction}

Interest rate models are mainly used to price and hedge bonds and bond options. One of the biggest assumptions is that interest rates $r$ are constant or, at least, known functions of time $r \equiv r(t)$. In reality this is far from the case. Many other securities that are influenced by interest rates have long duration. Their analysis in the presence of unpredictable interest rates is of crucial practical importance.

In this paper we will look at models for describing the stochastic behaviour of interest rates. Typically, the classical modesl are often based on assumptions that returns in the bond market are approximately normally distributed (Gaussian). For comprehensive introduction to interest rate modelling we refer to Bjork (1998), Brigo and Mercurio (2001), Filipović (2001), James and Webber (2000), Bingham and Kiesel (1998) and Rebonato (1996).

Empirical studies show that the Gaussian assumptions of the classical models do not hold in general. We wiil describe the Lévy-based models first introduced by Eberlein and Raible (1999). See also Raible (2000).

The main drawback of the Vasicek, the Cox-Ingersoll-Ross and other onefactor models lies in the fact that prices are explicit functions of the instantaneous 'spot' interest rate so that these models are unable to take the whole yield curve observed on the market into account in the price structure. Some authors have restored to a two-dimensional analysis to improve the models in terms of discrepencies between short and long rates. See, for example, Brennan and Schwartz (1979), Schaefer and Schwartz (1984), Courtadon (1982).

\footnotetext{
${ }^{1}$ Research is supported by NSERC grant RPG 312593
} 
These more complex models do not lead to explicit formulae and require the solution of partial differential equations. The general concept of multifactor affine-yield models is developed in Duffie and Kan (1994a,1994b). The reduction of affine-yield models to canonical versions is due to Dai and Singleton (2000). Maghsoodi (1996) provides a detailed study of the one-dimensional CIR equation when the parameters are time-varying. Although affine-yield models have simple bond price formulas, the prices for fixed income derivatives are more complicated. However, numerical solution of partial differential equations can be avoided by Fourier transform analysis (see Duffie, Pan and Singleton (2000)). There are many other common short rate models, such as Black, Derman and Toy (1990), Black and Karasinski (1991), and Longstaff and Schwartz (2001). An empirical comparison of various short rate models is provided by Chan et al. (1992).

Ho and Lee (1986) have proposed a discrete-time model describing the behaviour of the whole yield curve. The continuous-time model is based on the same idea and has been introduced by Heath, Jarrow and Morton (HJM) (1987). They considered forward interest rates. Filipovich (2001) examines the issue of making the yield curves generated by the HJM model consistent with the scheme used to generate the initial yield curve. Jara (2000) considers an HJM-type model but for interest rate futures rather than forward interest rates.

The use of a log-normal simple interest rate to price caps and floors was worked out by Miltersen, Sandman and Sondermann (1997). This idea was embedded in a full forward LIBOR term-structure model by Brace, Gatarek and Musiela (1997). Recently, a variation on forward LIBOR models has been developed for swaps markets (see Jamshidian (1990)). Term-structure models with jumps have been studied by Bjork, Kabanov and Rungaldier (1997), Das (1999), Das and Foresi (1996), Glasserman and Kou (2003), Galsserman and Merener (2003) and Shirakawa (1991). Three relatively recent books by authors with practical experience in term-structure modeling are Pelsser (2000), Brigo and Mercurio (2001) and Rebonato (2002).

In this paper, we show how to calculate the price of zero-coupon bonds for many models of $r(t)$ using change of time method, Swishchuk (2004, 2007). These models include, in particular, Ornshtein-Uhlenbeck (1930), Vasicek (1977), Cox-Ingersoll-Ross (1985), continuous-time GARCH, Ho-Lee (1986), Hull-White (1990) and Heath-Jarrrow-Morton (1992) models. In the same way, we can calculate the values of swaps, caps, floors and options on them, swaptions, captions and floortions, respectively. We also apply the change of time method to price the interest rate derivatives for the interest rates $r(t)$ described by various stochastic differential equations driven by $\alpha$-stable Lévy processes, see Sato (1999), Applebaum (2003), Schoutens (2003). The change of time method is convenient for presentation of a solution of a SDE. Sometimes one can not explicitly present the solution and uses numerical approaches. For example, there was no explicit solution's presentation for the CIR SDE (7). In Swishchuk (2004) the solution for this CIR SDE (7) was 
presented for the first time using change of time method. Applying change of time method we can use the presentation for the solution to calculate many financial derivatives, for example variance and volatility swaps, options for mean-reverting models, etc. (see Swishchuk (2004, 2007)). This paper incorporates the change of time method for pricing of interest rate derivatives.

The rest of the paper is organized as follows. In section 2 we give an overview of the basics of bond pricing. One-factor and multi-factor stochastic interest rate models, both Gaussian and Lévy, are introduced in section 3. The change of time method in Gaussian and Lévy settings is described in section 4. Solutions to all models considered in section 3 using change of time method are stated in section 5. Bond pricing in Gaussian and Lévy setting using both change of time and partial integro-differential equations is studied in section 6 . Various classes of interest rate derivatives, such as bond opotions, swaps, caps and floors, and options on them, swaptions, cvaptions and floortions, respectively, are introduced in section 7. Pricing of Gaussian and Lévy bond options is studied in section 8. Pricing of swaps, caps and floors for Gaussian and Lévy interest rate models is considered in section 9. Pricing of swaptions, captions and floortions in Gaussian and Lévy setting is studied in section 10. Section 11 concludes the paper. Appendix A contains one-factor and multi-factor Gaussian interest rate models and Appendix B gives the solutions to these models using change of time method.

\section{Basics of Bond Pricing}

\section{$2.1 \quad$ Zero-Coupon Bonds}

We begin with the subject of bond pricing. We do this under the assumption of a deterministic interest rate. This simplification allows us to discuss the effect of coupons on the prices of bonds and the appearence of the yield curve. A bond is a contract, paid for up-front, that yields a known amount on a known date in the future, the maturity date, $t=T$. The bond may also pay a known cash dividend ( the coupon) at fixed times during the life of the contract. If there is no coupon the bond is known as a zero-coupon bond. In this way, we call zero-coupon bond a security paying $\$ 1$ at a maturity date $T$ and we note $P(t, T)$ the value of this security at time $t$. We have $P(T, T)=1$

and in the world where the future is certain $P(t, T)=\exp \left(-\int_{t}^{T} r(s) d s\right)$, where $r(t)$ is the instanteneous interest rate.

\subsection{Bond Pricing with Known Interest Rates}

Let $V(t, T)$ be the value of the bond contract, $t<T$. If the interest rate $r(t)$ and coupon payment $K(t)$ are known functions of time, the bond price is also a function of time only: $V=V(t, T)$. If this bond pays the owner $Z$ at time $t=T$ then we know that $V(T, T)=Z$. We now derive an equation for 
the value of the bond at a time before maturity, $t<T$.

Suppose we hold one bond. Then arbitrage considerations lead us to the following equation:

$$
\frac{d V}{d t}+K(t)=r(t) V
$$

The right-hand side is the return we would have received had we converted our bond into cash at time t.

\subsection{Yield Curve}

Interest rates are not deterministic. For short-dated derivatives products such as options the errors associated with assuming a deterministic, or even constant, rate are small, typically $2 \%$. In dealing with products with a longer lifespan we must address the problem of random interest rate. The first step is to decide on a suitable measure for future values of interest rates, one that enables traders to communicate effectively about the same quantity. In the previous section we have seen a definition, (4), that gives an interest rate from bond price data but this relies on bond prices being differentiable with respect to the maturity date.

The yield curve is another measure of future values of interest rates. With the value of zero-coupon bonds $V(t, T)$ taken from real data, define

$$
Y(t, T)=-\frac{\log (V(t, T) / V(T, T))}{T-t},
$$

where $\mathrm{t}$ is the current time.

The yirld curve is the plot of $Y$ against time to maturity $T-t$. The dependence of the yield curve on the time to maturity is called the term structure of interest rate.

\subsection{The Short Rate}

The short rate $r(t):=Y(t, t)=\lim _{T \rightarrow t} Y(t, T)$ is the rate on instantaneous borrowing and lending. Historically, it was the short rate which was modelled as the basic process. In practice, this rate is stochastic and can fluctuate over time. Note that the short rate is actually a theoretical entity which does not exist in real life and can not be directly observed. A sum of 1 invested in the short rate at time zero and continuously rolled over, i.e., instantaneously reinvested, is called the money-market account. Its value

$S_{0}(t)=\exp \left[\int_{0}^{t} r(s) d s\right]$. If $r$ is deterministic and constant, $S_{0}(t)$ reduces to the classical bank account: $S_{0}(t)=B(t)=\exp [r t]$.

\subsection{The Instantaneous Forward Rate}

We define the instantaneous forward rate to be

$$
f(t, T):=-\frac{\partial \log P(t, T)}{\partial T} .
$$


The function $f(t, T)$ corresponds to the rate we can contract for at time $t$ on a riskless loan that begins at time $T$ and is returned an instant later. Since

$$
P(t, T)=\exp \left[-\int_{t}^{T} f(t, s) d s\right]
$$

zero-coupon bond prices and forward rates represent equivalent information. Note that the short rate $r(t)$ is contained in this forward rate structure since $r(t)=f(t, t)$.

\subsection{Stochastic Interest Rates}

For an uncertain future, one must think of the interest rate $r(t)$ in terms of a random or stochastic process (short rate). We consider a filtered probability space $\left(\Omega, \mathcal{F},\left(\mathcal{F}_{t}\right)_{0 \leq t \leq T}, P\right)$ and filtration $\mathcal{F}_{t}$ is the natural filtration of a standard Brownian motion $\left(W_{t}\right)_{0 \leq t \leq T}$ and $\mathcal{F}_{T}=\mathcal{F}$.

In view of our uncertainty about the future of the interest rate, it is natural to model it as a random variable. We should specify that $r$ is the interest rate received by the shortest possible deposit. The interest rate for the shortest posiible deposit is commonly called the spot rate.

We consider a model for the interest rate $r$ that is governed by a SDE of the form

$$
d r(t)=a(r, t) d t+b(r, t) d W(t)
$$

where $W$ is a standard Wiener process,

or

$$
d r(t)=a(r, t) d t+b(r, t) d L(t),
$$

where $L$ is a Lévy process, and $a$ and $b$ are 'good' functions.

A basic model for the behaviour of the stochastic process $r$ is the CIR model (Cox-Ingersoll-Ross (1985)). It is based on the CIR process:

$$
d r(t)=k(\theta-r(t)) d t+\gamma \sqrt{r} d W(t) .
$$

Under this model, the interest rate is mean reverting: it fluctuate around a long-term mean $\theta$. In the literature, this model was generalized in different ways. Well-known alternatives are the Vasicek and the Ho-Lee models, together with their extensions. We refer to Bjork (1998).

We consider a 'riskless' asset $S_{t}^{0}=\exp \left(\int_{0}^{t} r(s) d s\right)$, where $r(t)$ is an adapted process, such that $\int_{0}^{T}|r(s)| d s<+\infty$, almost surely. The risky assets are the zero-coupon bonds with maturity less or equal to the horizon $T$. For each $u \leq T$, we define adapted process $P(t, u), 0 \leq t \leq u$, satisfying $P(u, u)=1$, giving the price of the zero-coupon bond with maturity $u$ as a function of time. We note that $P(t, u)=E^{*}\left[\exp \left(-\int_{t}^{u} r(s) d s\right) \mid \mathcal{F}_{t}\right]$, where $E^{*}$ is an expectation with respect to the risk-neutral probability $P^{*}$. 
We note, that for each maturity $u$, there is an adapted process $\sigma(t, u), 0 \leq$ $t \leq u$, such that, on $[0, u]$,

$$
\frac{d P(t, u)}{P(t, u)}=(r(t)-\sigma(t, u) q(t)) d t+\sigma(t, u) d W(t)
$$

where $q(t)$ is an adapted process corresponding to 'risk premium' and $W(t)$ is a Wiener process under physical measure $P$. For example, for the Vasicek model $q(t)=-\lambda, \lambda \in R$, for the CIR model $q(t)=-\alpha \sqrt{r(t)}, \alpha \in R$.

The last formula is to be related with the equality $d S_{0}(t)=r(t) S_{0}(t) d t$, satisfied by the so-called riskless asset. It is the term in $d W(t)$ which makes the bond riskier.

Under risk-neutral probability $P^{*}$, the process $W^{*}(t)$ defined by $W^{*}(t):=$ $W(t)-\int_{0}^{t} q(s) d s$ is a standard Brownian motion (Girsanov theorem), and we have

$$
\frac{d P(t, u)}{P(t, u)}=r(t) d t+\sigma(t, u) d W^{*}(t) .
$$

(See Lamperton, Lapeyre (1993))

\section{Lévy Stochastic Interest Rate Models (SIRMs)}

Throughout the paper, we set $\left(\Omega, \mathcal{F},\left(\mathcal{F}_{t}\right)_{t \in R_{+}}, P\right)$ for a filtered probability space, Jacod and Shiryaev (1987).

In this section we give an overview on one-factor and multi-factor Lévy interest rate models.

\subsection{One-Factor Lévy SIRMs}

Definition 1. By LÉvy PROCESS we define a process with stationary and independent increments, Sato (1999), Applebaum (2003), Schoutens (2003).

Definiton 2. Let $\alpha \in(0,2]$. An $\alpha$-Stable LÉvy Process $L$ such that $L_{1}$ (or equivalently any $L_{t}$ ) has a strictly $\alpha$-stable distribution (i.e., $\left.L_{1} \equiv S_{\alpha}(\sigma, \beta, \nu)\right)$ for some $\alpha \in(0,2] \backslash\{1\}, \sigma \in R_{+}, \quad \beta \in[-1,1], \quad \nu=0$ or $\alpha=1, \quad \sigma \in R_{+}, \quad \beta=0, \quad \nu \in R$ ). (See Sato (1999)). We call $L$ a SYMMETRIC $\alpha$-STABLE LÉVY PROCESS if the distribution of $L_{1}$ is even symmetric $\alpha$-stable (i.e., $L_{1} \equiv S_{\alpha}(\sigma, 0,0)$ for some $\alpha \in(0,2], \quad \sigma \in R_{+}$.) A process $L$ is called $\left(T_{t}\right)_{t \in R_{+}}$-adapted if $L$ is constant on $\left[T_{t-}, T_{t}\right]$ for any $t \in R_{+}$.

$L(t)$ below is a symmetric $\alpha$-stable Lévy process with triplet $(\gamma, \sigma, \nu(d y))$. Similar to section 3.1 definitions of various processes defined via SDE driven by Brownian motion, we define below various processes via SDE driven by $\alpha$-stable Lévy process. 
1. Geometric $\alpha$-Stable Lévy Motion. $d r(t)=\mu r(t) d t+\sigma r(t) d L(t)$.

2. Ornstein-Uhlenbeck Process Driven by $\alpha$-Stable Lévy MoTION. $d r(t)=-\mu r(t) d t+\sigma d L(t)$.

3. Vasićé Process Driven by $\alpha$-Stable Lévy Motion. $d r(t)=$ $\mu(b-r(t)) d t+\sigma d L(t)$.

4. Continuous-Time GarCH Process Driven by $\alpha$-Stable Lévy PROCESS. $d r(t)=\mu(b-r(t)) d t+\sigma r(t) d L(t)$.

5. Cox-Ingersoll-Ross Process Driven by $\alpha$-Stable Lévy MoTION. $d r(t)=k(\theta-r(t)) d t+\gamma \sqrt{r(t)} d L(t)$.

6. Ho and Lee Process Driven by $\alpha$-Stable Lévy Motion. $d r(t)=\theta(t) d t+\sigma d L(t)$.

7. Hull and White Process Driven by $\alpha$-Stable Lévy Motion. $d r(t)=(a(t)-b(t) r(t)) d t+\sigma(t) d L(t)$

8. Heath, Jarrow and Morton Process Driven by $\alpha$-Stable LÉvy Motion. Define the forward interest rate $f(t, s)$, for $t \leq s$, characterized by the following equality $P(t, u)=\exp \left[-\int_{t}^{u} f(t, s) d s\right]$ for any maturity $u . f(t, s)$ represents the instanteneous interest rate at time $s$ as 'anticipated" by the market at time $t$. It is natural to set $f(t, t)=r(t)$. The process $f(t, u)_{0 \leq t \leq u}$ satisfies an equation $f(t, u)=f(0, u)+\int_{0}^{t} a(v, u) d v+$ $\int_{0}^{t} b(f(v, u)) d L(v)$, where the processes $a$ and $b$ are continuous.

\subsection{Multi-Factor Lévy SIRMs}

Multi-factor models driven by $\alpha$-stable Lévy motions can be obtained using various combinations of above-mentioned processes, see subsection 3.3. We give one example of two-factor continuous-time GARCH model driven by $\alpha$-stable Lévy motions.

$$
\left\{\begin{aligned}
d S(t) & =\mu(b(t)-S(t)) d t+\sigma S(t) d L^{1}(t) \\
d b(t) & =\xi b(t) d t+\eta b(t) d L^{2}(t),
\end{aligned}\right.
$$

where $L^{1}, L^{2}$ may be correlated, $\mu, \xi \in R, \sigma, \eta>0$.

Also, we can consider various combinations of models, presented above, i.e., mixed models containing Brownian and Lévy motions. For example,

$$
\left\{\begin{aligned}
d S(t) & =\mu(b(t)-S(t)) d t+\sigma S(t) d L(t) \\
d b(t) & =\xi b(t) d t+\eta b(t) d W(t)
\end{aligned}\right.
$$

where Brownian motion $W(t)$ and Lévy process $L(t)$ may be correlated.

Remark. Corresponding one-factor and multi-factor Gaussain interest rate models may be found in Appendix A.

\section{Change of Time Method}

Definition 3. A time Change is a right-continuous increasing $[0,+\infty]$ valued process $\left(T_{t}\right)_{t \in R_{+}}$such that $T_{t}$ is a stopping time for any $t \in R_{+}$. By 
$\hat{\mathcal{F}}_{t}:=\mathcal{F}_{T_{t}}$ we define the time-changed filtration $\left(\hat{\mathcal{F}}_{t}\right)_{t \in R_{+}}$. The INVERSE TIME CHANGe $\left(\hat{T}_{t}\right)_{t \in R_{+}}$is defined as $\hat{T}_{t}:=\inf \left\{s \in R_{+}: T_{s}>t\right\}$. (See Ikeda and Watanabe (1983)).

\subsection{Change of Time Method (CTM) for SDE driven by Brownian motion}

We consider the following SDE driven by a Brownian motion:

$$
d X(t)=a(t, X(t)) d W(t),
$$

where $W(t)$ is a Brownian motion and $a(t, X)$ is a continuous and measurable by $t$ and $X$ function on $[0,+\infty) \times R$.

The reason to consider this equation is the following one: if we solve the equation, then we can solve and more general equation with a drift $\beta(t, X)$ by drift transformation method or Girsanov transformation (see Ikeda and Watanabe (1981), Chapter 4, Section 4]). We use the following result to get the solution of the previous equation using change of time method.

Theorem. (Ikeda and Watanabe (1981), Chapter IV, Theorem 4.3) Let $\hat{W}(t)$ be an one-dimensional $\mathcal{F}_{t}$-Wiener process with $\hat{W}(0)=0$, given on a probability space $\left(\Omega, \mathcal{F},\left(\mathcal{F}_{t}\right)_{t \geq 0}, P\right)$ and let $X(0)$ be an $\mathcal{F}_{0}$-adopted random variable. Define a continuous process $V=V(t)$ by the equality

$$
V(t)=X(0)+\hat{W}(t) .
$$

Let $T_{t}$ be the change of time process (see Section 2.3):

$$
T_{t}=\int_{0}^{t} a^{-2}\left(T_{s}, X(0)+\hat{W}(s)\right) d s .
$$

If

$$
X(t):=V\left(\hat{T}_{t}\right)=X(0)+\hat{W}\left(\hat{T}_{t}\right)
$$

and $\hat{\mathcal{F}}_{t}:=\mathcal{F}_{\hat{T}_{t}}$, then there exists $\hat{\mathcal{F}}_{t^{-}}$adopted Wiener process $W=W(t)$ such that $(X(t), W(t))$ is a solution of $(2.1)$ on probability space $\left(\Omega, \mathcal{F}, \hat{\mathcal{F}}_{t}, P\right)$, where $\hat{T}_{t}$ is the inverse to $T_{t}$ time change.

\subsection{Change of Time Method (CTM) for SDE driven by Lévy motion}

We denote by $L_{\text {a.s. }}^{\alpha}$ the family of all real measurable $\mathcal{F}_{t}$-adapted processes $a$ on $\Omega \times[0,+\infty)$ such that for every $T>0, \int_{0}^{T}|a(t, \omega)|^{\alpha} d t<+\infty$ a.s. We consider the following SDE driven by a Lévy motion:

$$
d X(t)=a(t, X(t)) d L(t),
$$


with the same reason as above for SDE with a Brownian motion as a driven process.

We are going to use the following result to get the solutions of SDE driven by Lévy processes.

Theorem. (Rosinski and Woyczynski (1986), Theorem 3.1., p.277). Let $a \in L_{a . s .}^{\alpha}$ be such that $T(u):=\int_{0}^{u}|a|^{\alpha} d t \rightarrow+\infty$ a.s. as $u \rightarrow+\infty$. If $\hat{T}(t):=\inf \{u: T(u)>t\}$ and $\hat{\mathcal{F}}_{t}=\mathcal{F}_{\hat{T}(t)}$, then the time-changed ctochastic

integral $\hat{L}(t)=\int_{0}^{\hat{T}(t)} a d L(t)$ is an $\hat{\mathcal{F}}_{t}-\alpha$-stable Lévy process, where $L(t)$ is $\mathcal{F}_{t^{-}}$adapted and $\mathcal{F}_{t}-\alpha$-stable Lévy process. Consequently, a.s. for each $t>0$ $\int_{0}^{t} a d L=\hat{L}(T(t))$, i.e., the stochastic integral with respect to a $\alpha$-stable Lévy process is nothing but another $\alpha$-stable Lévy process with randomly changed time scale.

\section{Solutions Lévy SIRMs using CTM}

\subsection{Solution of One-Factor Lévy SIRMs Using CTM}

Below we give the solutions to some one-factor Lévy IRM decribed by SDE driven by $\alpha$-stable Lévy process. $L(t)$ below is a symmetric $\alpha$-stable Lévy process, and $\hat{L}$ is a $\left(\hat{T}_{t}\right)_{t \in R_{+}}$-adapted symmetric $\alpha$-stable Levy process on $\left.\left(\Omega, \mathcal{F},\left(\hat{\mathcal{F}}_{t}\right)_{t \in R_{+}}, P\right)\right)$.

1. Geometric $\alpha$-Stable LÉvy Motion. $d r(t)=\mu r(t) d t+\sigma r(t) d L(t)$. Solution $r(t)=e^{\mu t}\left[r(0)+\hat{L}\left(\hat{T}_{t}\right)\right]$, where $\hat{T}_{t}=\sigma^{\alpha} \int_{0}^{t}\left[r(0)+\hat{L}\left(\hat{T}_{s}\right)\right]^{\alpha} d s$.

2. Ornstein-Uhlenbeck Process Driven By $\alpha$-Stable Lévy MoTION. $d r(t)=-\mu r(t) d t+\sigma d L(t)$. Solution $r(t)=e^{-\mu t}\left[r(0)+\hat{L}\left(\hat{T}_{t}\right)\right]$, where $\hat{T}_{t}=\sigma^{\alpha} \int_{0}^{t}\left(e^{\mu s}\left[r(0)+\hat{L}\left(\hat{T}_{s}\right)\right]\right)^{\alpha} d s$.

3. VAsićek Process Driven By $\alpha$-Stable Lévy Motion. $d r(t)=$ $\mu(b-r(t)) d t+\sigma d L(t)$. Solution $r(t)=e^{-\mu t}\left[r(0)-b+\hat{L}\left(\hat{T}_{t}\right)\right]$, where $\hat{T}_{t}=$ $\sigma^{\alpha} \int_{0}^{t}\left(e^{\mu s}\left[r(0)-b+\hat{L}\left(\hat{T}_{s}\right)\right]+b\right)^{\alpha} d s$.

4. Continuous-Time GARCH Process Driven By $\alpha$-STABle LÉvy PROCESS. $d r(t)=\mu(b-r(t)) d t+\sigma r(t) d L(t)$. Solution $r(t)=e^{-\mu t}(r(0)-b+$ $\left.\hat{L}\left(\hat{T}_{t}\right)\right)+b$, where $\hat{T}_{t}=\sigma^{\alpha} \int_{0}^{t}\left[r(0)-b+\hat{L}\left(\hat{T}_{s}\right)+e^{\mu s} b\right]^{\alpha} d s$.

5. Cox-Ingersoll-Ross Process Driven By $\alpha$-Stable Lévy MoTION. $d r(t)=k\left(\theta^{2}-r(t)\right) d t+\gamma \sqrt{r(t)} d L(t)$. Solution $r^{2}(t)=e^{-k t}\left[r_{0}^{2}-\theta^{2}+\right.$ $\left.\hat{L}\left(\hat{T}_{t}\right)\right]+\theta^{2}$, where $T_{t}=\gamma^{-\alpha} \int_{0}^{t}\left[e^{k T_{s}}\left(r_{0}^{2}-\theta^{2}+\hat{W}(s)\right)+\theta^{2} e^{2 k T_{s}}\right]^{-\alpha / 2} d s$.

6. Ho and Lee Process Driven by $\alpha$-Stable Lévy Motion. $d r(t)=\theta(t) d t+\sigma d L(t)$. Solution $r(t)=r(0)+\hat{L}\left(\sigma^{2} t\right)+\int_{0}^{t} \theta(s) d s$.

7. Hull And White Driven By $\alpha$-Stable Lévy Motion. $d r(t)=$ $(a(t)-b(t) r(t)) d t+\sigma(t) d L(t)$. Solution $r(t)=\exp \left[-\int_{0}^{t} b(s) d s\right]\left[r(0)-\frac{a(s)}{b(s)}+\right.$ $\left.\hat{L}\left(\hat{T}_{t}\right)\right]$, where $\hat{T}_{t}=\int_{0}^{t} \sigma^{2}(s)\left[r(0)-\frac{a(s)}{b(s)}+\hat{L}\left(\hat{T}_{s}\right)+\exp \left[\int_{0}^{s} b(u) d u\right] \frac{a(s)}{b(s)}\right] d s$.

8. Heath, Jarrow and Morton Driven by $\alpha$-Stable Lévy MoTION. $f(t, u)=f(0, u)+\int_{0}^{t} a(v, u) d v+\int_{0}^{t} b(f(v, u)) d L(v)$. Solution $f(t, u)=$ $f(0, u)+\hat{L}\left(\hat{T}_{t}\right)+\int_{0}^{t} a(v, u) d v$, where $\hat{T}_{t}=\int_{0}^{t} b^{2}\left(f(0, u)+\hat{L}\left(\hat{T}_{s}\right)+\int_{0}^{s} a(v, u) d v\right) d s$. 


\subsection{Solution of Multi-Factor Lévy SIRMs Using CTM}

Solution of multi-factor models driven by $\alpha$-stable Lévy motions can be obtained using various combinations of solutions of the above-mentioned processes, see subsection 5.3, and CTM. We give one example of two-factor continuous-time GARCH model driven by $\alpha$-stable Lévy motions:

$$
\left\{\begin{aligned}
d r(t) & =\mu(b(t)-r(t)) d t+\sigma r(t) d L^{1}(t) \\
d b(t) & =\xi b(t) d t+\eta b(t) d L^{2}(t),
\end{aligned}\right.
$$

where $L^{1}, L^{2}$ may be correlated, $\mu, \xi \in R, \sigma, \eta>0$. Solution, using CTM for the first and the second equations, subsection 5.3: $r(t)=e^{-\mu t}[r(0)-$ $\left.e^{\xi t}\left(b(0)+\hat{L}^{2}\left(\hat{T}_{t}^{2}\right)\right)+\hat{L}^{1}\left(\hat{T}_{t}^{1}\right)\right]+e^{\xi t}\left[b(0)+\hat{L}^{2}\left(T_{t}^{2}\right)\right]$, where $\hat{T}^{i}$ are defined in 4 . $(i=1)$ and 1. $(i=2)$, respectevely, subsection 5.3.

Remark. Solutions of one-factor and multi-factor Gaussian SIRMs may be found in Appendix B.

\section{Bond Pricing}

Pricing a bond is harder than pricing an option even in Gaussian case, since there is no underlying asset with which to hedge: you can not buy an interest rate of, say, $15 \%$. The only alternative is to hedge in Gaussian case is the case with bonds of different maturity dates. To calculate the value of a European options with maturity $\theta$ on the zero-coupon bond with maturity equal to $T$ we could proceed as follows. If it is a call with strike price $K$, the value of the option at time $\theta$ is $\max (P(\theta, T)-K), 0)$ and it seems reasonable to hedge this call with a portfolio of riskless asset $S_{t}^{0}$ and zero-coupon bond $P(\theta, T)$ with maturity $T$. Using the classical arguments (and using the money-market account as numeraire), the price at time 0 of a European call option on a bond is given by

$$
V(\theta, T, r)=E^{*}\left[\frac{1}{S_{0}(t)} \max (P(\theta, T)-K, 0)\right],
$$

where $E^{*}$ is the expectation under the risk-neutral measure, $S_{0}(t)=\exp \left[\int_{0}^{t} r(s) d s\right]$ and $P(\theta, T)$ is defined in section 2.6.

\subsection{Gaussian Bond Pricing for One-Factor SIRMs via CTM}

The solution of the SDE (8) or (9) can be written in the form

$$
\begin{array}{r}
P(t, T)=P(0, T) \exp \left[\int_{0}^{T} r(s) d s\right] \frac{\exp \left[\int_{0}^{t} \sigma(s, T) d \hat{W}(s)\right]}{E\left\{\exp \left[\int_{0}^{t} \sigma(s, T) d \hat{W}(s)\right]\right\}} \\
=P(0, T) \exp \left[\int_{0}^{T} r(s) d s\right] \exp \left[\int_{0}^{t} \sigma(s, T) d \hat{W}(s)-\frac{1}{2} \int_{0}^{t} \sigma(s, T)^{2} d s\right],
\end{array}
$$


where $r(t)$ has one of the representations in section 5.1.

We see that the $\log$ returns under risk-neutral measure approximately follow a Normally distributed random variable.

Remark. Empirical studies (see, for example, Raible (2000)) show that the normality assumption does not reflect reality. Empirically observed log returns of bonds turn out to have leptokurtic distribution.

\subsection{Gaussian Bond Pricing for One-Factor SIRMs via PDE}

Consider $V(t, T, r)$-bond price at time $t$, where interest rate $r(t)$ follows the following SDE (in general form)

$$
d r(t)=a(r, t) d t+b(r, t) d W(t) .
$$

For example, for GBM, $a=\mu r$ and $b=\sigma r$, for OU process, $a=-\mu r$ and $b=\sigma$, and so on (see section 3.1).

The zero-coupon bond pricing equation is

$$
\frac{\partial V}{\partial t}+\frac{1}{2} b^{2} \frac{\partial^{2} V}{\partial r^{2}}+(a-\lambda b) \frac{\partial V}{\partial r}-r V=0,
$$

where the function $\lambda$ is often called the market price of risk. The final condition is

$$
V(T, T, r)=Z \text {. }
$$

This PDE may be solved approximately by standard numerical methods, see, for example, Wilmott, Howison and Dewynne (1995).

Remark. To price options on bonds with coupon, the reader is referred to Jamshidian (1989) and El Karoui and Rochet (1989).

\subsection{Lévy Bond Pricing for One-Factor SIRMs via CTM and Fourier Transform}

We model the zero-coupon bond price with the following process (see Eberlein and Raible (1999), Raible (2000)):

$$
P(t, T)=P(0, T) \exp \left[\int_{0}^{T} r(s) d s\right] \frac{\exp \left[\int_{0}^{t} \sigma(s, T) d L(s)\right]}{E\left\{\exp \left[\int_{0}^{t} \sigma(s, T) d L(s)\right]\right\}},
$$

where $r(t)$ has one of the representations in section 5.3.

Eberlein and Raible (1999) derived the bond price process in the form

$$
P(t, T)=P(0, T) \exp \left[\int_{0}^{T} r(s) d s\right] \frac{\exp \left[\int_{0}^{t} \sigma(s, T) d L(s)\right]}{\exp \left[\int_{0}^{t} \theta(\sigma(s, T)) d L(s)\right]},
$$

where $\theta(u):=\log (E[\exp (u L(1))])$ denotes the logarithm of the momentgenerating function of the Lévy process at time 1 . For example, in the 
classical Gaussian model we choose $\theta(u)=u^{2} / 2$ and $L(s)=W(s)$. We note, that we know the expressions for $r(t)$ in the above formula for many SIRMs, see section 5.3.

Except when $L(t)$ is a Poisson or a Brownian motion, our Lévy market model is an incomplete model. It means that there are many different equivalent martingales measures to choose. In general this leads to many different possible prices for European options or bond options, etc.

One of the way to price bond is to use for the $P^{*}$ in (19) the Esscher transform equivalent martingale measure. Following Gerber and Shiu (1994), we can by using the so-called Esscher transform find in some cases at least one equaivalent martingale measure $P^{*}$. Let $f(t, x)$ be the density of our model's (real world, i.e. under $P$ ) distribution of $L(t)$. For some real number $\theta \in\left\{\theta \in R \mid \int_{-\infty}^{+\infty} \exp (\theta y) f(t, y) d y<+\infty\right\}$ we can define a new density

$$
f^{(\theta)}(t, x)=\frac{\exp (\theta x) f(t, x)}{\int_{-\infty}^{+\infty} \exp (\theta y) f(t, y) d y} .
$$

In order to assume finiteness of the expectation in the denominator above in the case of general Lévy processes, we assume that

$$
\int_{\{|x|>1\}} \exp [v x] \nu(d x)<\infty, \quad \text { for } \quad|v|<(1+\epsilon) M
$$

where $\epsilon>0$ and $M$ is such that $0 \leq \sigma(s, T) \leq M$ (a.s.) (see (8) and (9)) for $0 \leq s \leq T$ and $\nu(d x)$ is the Lévy measure of $L_{1}$. Typical choices of this Lévy process are the variance gamma, the normal inverse Gaussian, the generalized hyperbolic, the Meixner or CGMY processes (see Schoutens (2003)). Another way to price bond is to consider characteristic function (or Fourier transform), if it is known, of the risk-neutral log returns (see Carr and Madan (1998)). We note, that if we know the explicit expression for $r(t)$ (see section 5.3), then we can find the characteristic function of the risk-neutral bond price.

\subsection{Lévy Bond Pricing via PIDE}

One more way to price bond is to consider the solution of a partial differential integral equation with boundary condition, all in terms of the triplet of Lévy characteristics $\left[\gamma, \sigma, \nu^{P^{*}}(d y)\right]$ of the Lévy process under the risk-neutral measure $P^{*}$.

Consider $V(t, T, r)$-bond price at time $t$, where interest rate $r(t)$ follows the following SDE (in general form)

$$
d r(t)=a(r, t) d t+b(r, t) d L(t)
$$

$L(t)$ is a Lévy process.

For example, for GBM, $a=\mu r$ and $b=\sigma r$, for OU process, $a=-\mu r$ and $b=\sigma$, and so on (see section 3.1). 
The zero-coupon bond pricing eqaution is

$$
\begin{aligned}
\frac{\partial V}{\partial t} & +\frac{1}{2} b^{2} \sigma^{2} \frac{\partial^{2} V}{\partial r^{2}}+(a+\gamma b-\lambda b \sigma) \frac{\partial V}{\partial r} \\
& +\int_{-\infty}^{+\infty}\left[V(t, r+b y)-V(t, r)-b y \frac{\partial V(t, r)}{\partial r}\right] \nu(d y)-r V=0
\end{aligned}
$$

where $\lambda$ is the market price of risk. The final condition is

$$
V(T, T, r)=Z
$$

This PDIE is the analogue of the famous Black-Scholes PDE and follows from the Feynman-Kac formula for Lévy processes.

Since there is no closed solution in general we must resort to approximate methods. We may pose the PIDE in a form that becomes amenable to a solution using the finite difference method. A number of schemes is discussed in Duffy (2005). He considers a) implicit and explicit methods, b) implicitexplicit Runge-Kutta methods, c) operator splitting (method of fractional steps) and d) splitting in conjuction with predictor-corrector methods. See also Cont and Voltchkova (2003, 2005).

Remark. European call option price for the stock price that follows exponential Lévy process was derived by Nualart and Schoutens (2001) and Raible (2000).

\section{Interest Rate Derivatives}

There is a large number of different interest rate derivative products. Here, we consider options, swaps, caps and floors. Having valued swaps, caps and floors we can value options on these instruments: swaptions, captions, floortions.

A bond option is the option on bond ans is identical to an equity option except that the underlying asset is a bond.

Both European and American versions exist.

An interest rate swap is an agreement between two parties to exchange the interest rate payments on a certain amount, the principal, for a certain length of time.

One party $A$ pays the other $B$ a fixed rate of interest rate in return for a variable interest rate payment. For example, $A$ pays $10 \%$ of $\$ 1,000,000$ p.a. to $B$ and $B$ pays $r$ (variable) of the same amount to $A$. This agreement is to last for three years, say.

A cap is a loan at the floating interest rate but with the proviso that the interest rate charged is quaranteed not to exceed a specified value, the cap, which is denote by $r^{*}$.

A floor is similar to a cap except that the interest rate does not go below $r^{*}$.

Swaption is an option on swap. Caption is an option on cap. Floortion is an option on floor. 


\section{Pricing of Gaussian and Lévy Bond Op- tions}

\subsection{Pricing of Gaussian Bond Options}

Let interest rate $r(t)$ follows the following SDE (in general form)

$$
d r(t)=a(r, t) d t+b(r, t) d W(t)
$$

where $W(t)$ is a standard Wiener process.

Consider the European call bond option, with exercise price $K$ and expity date $T$, on a zero-coupon bond with maturity date $T_{B} \geq T$.

To find the value of the call option on bond (to buy a bond) we proceed with the following steps:

1. To find the value of the bond: $V_{B}\left(r, t ; T_{B}\right)$, that satisfies the following PDE:

$$
\frac{\partial V_{B}}{\partial t}+\frac{1}{2} b^{2} \frac{\partial^{2} V_{B}}{\partial r^{2}}+(a-\lambda b) \frac{\partial V_{B}}{\partial r}-r V_{B}=0 .
$$

with the final condition

$$
V_{B}\left(r, T_{B} ; T_{B}\right)=Z
$$

2.Let $C_{B}(r, t)$ be the value of the call option on this bond. Since $C_{B}$ also depends on the random walk $r(t)$, it must satisfy equation (1) too:

$$
\frac{\partial C_{B}}{\partial t}+\frac{1}{2} b^{2} \frac{\partial^{2} C_{B}}{\partial r^{2}}+(a-\lambda b) \frac{\partial C_{B}}{\partial r}-r C_{B}=0
$$

with the final condition

$$
C_{B}(r, T)=\max \left(V_{B}\left(r, T ; T_{B}\right)-K, 0\right) .
$$

Remark. These PDEs can be solved numerically using standard methods, see Wilmott, Howison and Dewynne (1995).

\subsection{Pricing of Lévy Bond Options}

Let interest rate $r(t)$ follows the following SDE (in general form)

$$
d r(t)=a(r, t) d t+b(r, t) d L(t)
$$

where $L(t)$ is a Lévy process.

Consider the European Call Bond Option, with exercise price $K$ and expity date $T$, on a zero-coupon bond with maturity date $T_{B} \geq T$.

To find the value of the call option on bond (to buy a bond) we proceed with the following steps:

1. To find the value of the bond: $V_{B}\left(r, t ; T_{B}\right)$, that satisfies the following PIDE: 


$$
\begin{aligned}
\frac{\partial V_{B}}{\partial t} & +\frac{1}{2} b^{2} \sigma^{2} \frac{\partial^{2} V_{B}}{\partial r^{2}}+(a+b \gamma-\lambda b \sigma) \frac{\partial V_{B}}{\partial r} \\
& +\int_{-\infty}^{+\infty}\left[V_{B}(t, r+b y)-V_{B}(t, r)-b y \frac{\partial V_{B}(t, r)}{\partial r}\right] \nu(d y)-r V_{B}=0
\end{aligned}
$$

with the final condition

$$
V_{B}\left(r, T_{B} ; T_{B}\right)=Z \text {. }
$$

2. Let $C_{B}(r, t)$ be the value of the call option on this bond. Since $C_{B}$ also depends on the random walk $r(t)$, it must satisfy equation (27) too:

$$
\begin{aligned}
\frac{\partial C_{B}}{\partial t} & +\frac{1}{2} b^{2} \sigma^{2} \frac{\partial^{2} C_{B}}{\partial r^{2}}+(a+b \gamma-\lambda b \sigma) \frac{\partial C_{B}}{\partial r} \\
& +\int_{-\infty}^{+\infty}\left[C_{B}(t, r+b y)-C_{B}(t, r)-b y \frac{\partial C_{B}(t, r)}{\partial r}\right] \nu(d y)-r C_{B}=0 .
\end{aligned}
$$

with the final condition

$$
C_{B}(r, T)=\max \left(V_{B}\left(r, T ; T_{B}\right)-K, 0\right)
$$

Remark. One of the approach to solve this PDIE could be numerical using different finite difference methods, see Duffy (2005).

\section{Pricing of Swaps, Caps and Floors}

\subsection{Pricing of Swaps, Caps and Floors for Gaussian IRMs}

Let interest rate $r(t)$ follows the following SDE (in general form)

$$
d r(t)=a(r, t) d t+b(r, t) d W(t)
$$

where $W(t)$ is a standard Wiener process.

Pricing of Swaps. We consider to value such swaps in general. Suppose that $A$ pays the interest on an amount $Z$ to $\mathrm{B}$ at a fixed rate $r^{*}$ and $B$ pays interest to $A$ at the floating rate $r$. These payments continue until time $T_{S}$. Denote the value of this swap to A by $Z V_{S}(r, t)$. We note, that in a time-step dt A receives $\left(r-r^{*}\right) Z d t$. If we think of this payment as being similar to a coupon payment on a simple bond then we find that:

$$
\frac{\partial V_{S}}{\partial t}+\frac{1}{2} b^{2} \frac{\partial^{2} V_{S}}{\partial r^{2}}+(a-\lambda b) \frac{\partial V_{S}}{\partial r}-r V_{S}+\left(r-r^{*}\right)=0
$$

with the final condition

$$
V_{S}\left(r, T_{S}\right)=0
$$

We note, that $r$ can be greater or less than $r^{*}$ and so $V_{S}(r, t)$ need not be positive. 
Pricing of Caps. The loan of $\mathrm{Z}$ is to be paid back at time $T_{C}$. The value of the capped loan, $Z V_{C}(r, t)$ satisfies the following PDE:

$$
\frac{\partial V_{C}}{\partial t}+\frac{1}{2} b^{2} \frac{\partial^{2} V_{C}}{\partial r^{2}}+(a-\lambda b) \frac{\partial V_{C}}{\partial r}-r V_{C}+\min \left(r, r^{*}\right)=0 .
$$

with the final condition

$$
V_{C}\left(r, T_{C}\right)=1 .
$$

Pricing of Floors. The value of the floored loan, $Z V_{F}(r, t)$, satisfies the following PDE:

$$
\frac{\partial V_{F}}{\partial t}+\frac{1}{2} b^{2} \frac{\partial^{2} V_{F}}{\partial r^{2}}+(a-\lambda b) \frac{\partial V_{F}}{\partial r}-r V_{F}+\max \left(r, r^{*}\right)=0 .
$$

with the final condition

$$
V_{F}\left(r, T_{F}\right)=1,
$$

where $T_{F}$ is an expiry time for floor.

Remark. These PDE can be solved numerically using standard methods, see Wilmott, Howison and Dewynne (1995).

\subsection{Pricing of Swaps, Caps and Floors for Lévy IRMs}

Consider $V(r, t)$-bond price at time $t$, where interest rate $r(t)$ follows the following SDE (in general form)

$$
d r(t)=a(r, t) d t+b(r, t) d L(t),
$$

$L(t)$ is a Lévy process.

Pricing of Swaps. We consider to value such swaps in general. Suppose that $A$ pays the interest on an amount $Z$ to $\mathrm{B}$ at a fixed rate $r^{*}$ and $B$ pays interest to $A$ at the floating rate $r$. These payments continue until time $T_{S}$. Denote the value of this swap to A by $Z V_{S}(r, t)$. We note, that in a time-step dt A receives $\left(r-r^{*}\right) Z d t$. If we think of this payment as being similar to a coupon payment on a simple bond then we find that:

$$
\begin{aligned}
\frac{\partial V_{S}}{\partial t} & +\frac{1}{2} b^{2} \sigma^{2} \frac{\partial^{2} V_{S}}{\partial r^{2}}+(a+b \gamma-\lambda b \sigma) \frac{\partial V_{S}}{\partial r} \\
& +\int_{-\infty}^{+\infty}\left[V_{S}(t, r+b y)-V_{S}(t, r)-b y \frac{\partial V_{S}(t, r)}{\partial r}\right] \nu(d y) \\
& -r V_{S}+\left(r-r^{*}\right)=0 .
\end{aligned}
$$

with the final condition

$$
V_{S}\left(r, T_{S}\right)=0 .
$$

We note, that $r$ can be greater or less than $r^{*}$ and so $V_{S}(r, t)$ need not be positive.

Pricing of Caps. The loan of $\mathrm{Z}$ is to be paid back at time $T_{C}$. The value of the capped loan, $Z V_{C}(r, t)$ satisfies the following PIDE:

$$
\begin{aligned}
\frac{\partial V_{C}}{\partial t} & +\frac{1}{2} b^{2} \sigma^{2} \frac{\partial^{2} V_{C}}{\partial r^{2}}+(a+b \gamma-\lambda b \sigma) \frac{\partial V_{C}}{\partial r} \\
& +\int_{-\infty}^{+\infty}\left[V_{C}(t, r+b y)-V_{C}(t, r)-b y \frac{\partial V_{C}(t, r)}{\partial r}\right] \nu(d y) \\
& -r V_{C}+\min \left(r, r^{*}\right)=0 .
\end{aligned}
$$


with the final condition

$$
V_{C}\left(r, T_{C}\right)=1 .
$$

Pricing of Floors. The value of the floored loan, $Z V_{F}(r, t)$, satisfies the following PDE:

$$
\begin{aligned}
\frac{\partial V_{F}}{\partial t} & +\frac{1}{2} b^{2} \sigma^{2} \frac{\partial^{2} V_{F}}{\partial r^{2}}+(a+b \gamma-\lambda b \sigma) \frac{\partial V_{F}}{\partial r} \\
& +\int_{-\infty}^{+\infty}\left[V_{F}(t, r+b y)-V_{F}(t, r)-b y \frac{\partial V_{F}(t, r)}{\partial r}\right] \nu(d y) \\
& -r V_{F}+\max \left(r, r^{*}\right)=0 .
\end{aligned}
$$

with the final condition

$$
V_{F}\left(r, T_{F}\right)=1,
$$

where $T_{F}$ is an expiry time for floor.

Remark. One of the approach to solve thess PDIEs could be numerical using different finite difference methods, see Duffy (2005).

\section{Pricing of Swaptions, Captions and Floor- tions}

\subsection{Pricing of Swaptions, Captions and Floortions for Gaussian IRMs}

Let interest rate $r(t)$ follows the following SDE (in general form)

$$
d r(t)=a(r, t) d t+b(r, t) d W(t)
$$

where $W(t)$ is a standard Wiener process.

Pricing of Swaptions. Consider European Swap Call Option, option to buy this swap (a call swaption) for an amount $K$ at time $T<T_{S}$, where $T_{S}$ is an expiry time for swap with value $V_{S}(r, t), t \leq T_{S}$. Thus, this value $V_{S}$ satisfies the following PDE (see (32)):

$$
\frac{\partial V_{S}}{\partial t}+\frac{1}{2} b^{2} \frac{\partial^{2} V_{S}}{\partial r^{2}}+(a-\lambda b) \frac{\partial V_{S}}{\partial r}-r V_{S}+\left(r-r^{*}\right)=0 .
$$

with the final condition

$$
V_{S}\left(r, T_{S}\right)=0 .
$$

Then the value $C_{S}(r, t)$ of this call swap option (call swaption) satisfies the following PDE:

$$
\frac{\partial C_{S}}{\partial t}+\frac{1}{2} b^{2} \frac{\partial^{2} C_{S}}{\partial r^{2}}+(a-\lambda b) \frac{\partial C_{S}}{\partial r}-r C_{S}=0 .
$$

with the final condition

$$
C_{S}(r, T)=\max \left(V_{S}(r, T)-K, 0\right) .
$$


We solve for the value of the swap first and then use this value as the final data for the value of the swaption.

Pricing of Captions. Consider European Cap Call Option, option to buy this cap (a call caption) for an amount $K$ at time $T<T_{C}$, where $T_{C}$ is an expiry time for cap with value $V_{C}(r, t), t \leq T_{C}$. Thus, this value $V_{C}$ satisfies the following PDE (see (33)):

$$
\frac{\partial V_{C}}{\partial t}+\frac{1}{2} b^{2} \frac{\partial^{2} V_{C}}{\partial r^{2}}+(a-\lambda b) \frac{\partial V_{C}}{\partial r}-r V_{C}+\min \left(r, r^{*}\right)=0 .
$$

with the final condition

$$
V_{C}\left(r, T_{C}\right)=1
$$

Then the value $C_{C}(r, t)$ of this call cap option (call caption) satisfies the following PDE:

$$
\frac{\partial C_{C}}{\partial t}+\frac{1}{2} b^{2} \frac{\partial^{2} C_{C}}{\partial r^{2}}+(a-\lambda b) \frac{\partial C_{C}}{\partial r}-r C_{C}=0
$$

with the final condition

$$
C(r, T)=\max \left(V_{C}(r, T)-K, 0\right) .
$$

We solve for the value of the cap first and then use this value as the final data for the value of the caption.

Pricing of Floortions. Consider European Floor Call Option, option to buy this floor (a call floortion) for an amount $K$ at time $T<T_{F}$, where $T_{F}$ is an expiry time for floor with value $V_{F}(r, t), t \leq T_{F}$. Thus, this value $V_{F}$ satisfies the following PDE (see (34)):

$$
\frac{\partial V_{F}}{\partial t}+\frac{1}{2} b^{2} \frac{\partial^{2} V_{F}}{\partial r^{2}}+(a-\lambda b) \frac{\partial V_{F}}{\partial r}-r V_{F}+\min \left(r, r^{*}\right)=0 .
$$

with the final condition

$$
V_{C}\left(r, T_{F}\right)=1
$$

Then the value $C_{F}(r, t)$ of this call floor option (call floortion) satisfies the following PDE:

$$
\frac{\partial C_{F}}{\partial t}+\frac{1}{2} b^{2} \frac{\partial^{2} C_{F}}{\partial r^{2}}+(a-\lambda b) \frac{\partial C_{F}}{\partial r}-r C_{F}=0
$$

with the final condition

$$
C_{F}(r, T)=\max \left(V_{F}(r, T)-K, 0\right) .
$$

We solve for the value of the floor first and then use this value as the final data for the value of the floortion.

Remark. These PDE can be solved numerically using standard methods, see Wilmott, Howison and Dewynne (1995). 


\subsection{Pricing of Swaptions, Captions and Floortions for Lévy IRMs}

Consider $V(r, t)$-bond price at time $t$, where interest rate $r(t)$ follows the following SDE (in general form)

$$
d r(t)=a(r, t) d t+b(r, t) d L(t),
$$

$L(t)$ is a Lévy process.

Pricing of Swaptions. Consider European Swap Call Option, option to buy this swap (a call swaption) for an amount $K$ at time $T<T_{S}$, where $T_{S}$ is an expiry time for swap with value $V_{S}(r, t), t \leq T_{S}$. Thus, this value $V_{S}$ satisfies the following PIDE (see (36)):

$$
\begin{aligned}
\frac{\partial V_{S}}{\partial t} & +\frac{1}{2} b^{2} \sigma^{2} \frac{\partial^{2} V_{S}}{\partial r^{2}}+(a+b \gamma-\lambda b \sigma) \frac{\partial V_{S}}{\partial r} \\
& +\int_{-\infty}^{+\infty}\left[V_{S}(t, r+b y)-V_{S}(t, r)-b y \frac{\partial V_{S}(t, r)}{\partial r}\right] \nu(d y) \\
& -r V_{S}+\left(r-r^{*}\right)=0 .
\end{aligned}
$$

with the final condition

$$
V_{S}\left(r, T_{S}\right)=0
$$

Then the value $C_{S}(r, t)$ of this call swap option (call swaption) satisfies the following PIDE:

$$
\begin{aligned}
\frac{\partial C_{S}}{\partial t} & +\frac{1}{2} b^{2} \sigma^{2} \frac{\partial^{2} C_{S}}{\partial r^{2}}+(a+b \gamma-\lambda b \sigma) \frac{\partial C_{S}}{\partial r} \\
& +\int_{-\infty}^{+\infty}\left[C_{S}(t, r+b y)-C_{S}(t, r)-b y \frac{\partial C_{S}(t, r)}{\partial r}\right] \nu(d y)-r C_{S}=0
\end{aligned}
$$

with the final condition

$$
C_{S}(r, T)=\max \left(V_{S}(r, T)-K, 0\right) .
$$

We solve for the value of the swap first and then use this value as the final data for the value of the swaption.

Pricing of Captions. Consider European Cap Call Option, option to buy this cap (a call caption) for an amount $K$ at time $T<T_{C}$, where $T_{C}$ is an expiry time for cap with value $V_{C}(r, t), t \leq T_{C}$. Thus, this value $V_{C}$ satisfies the following PIDE (see (37)):

$$
\begin{aligned}
\frac{\partial V_{C}}{\partial t} & +\frac{1}{2} b^{2} \sigma^{2} \frac{\partial^{2} V_{C}}{\partial r^{2}}+(a+b \gamma-\lambda b \sigma) \frac{\partial V_{C}}{\partial r} \\
& +\int_{-\infty}^{+\infty}\left[V_{C}(t, r+b y)-V_{C}(t, r)-b y \frac{\partial V_{C}(t, r)}{\partial r}\right] \nu(d y) \\
& -r V_{C}+\min \left(r, r^{*}\right)=0
\end{aligned}
$$

with the final condition

$$
V_{C}\left(r, T_{C}\right)=1 \text {. }
$$

Then the value $C_{C}(r, t)$ of this call cap option (call caption) satisfies the following PDE:

$$
\begin{aligned}
\frac{\partial C_{C}}{\partial t} & +\frac{1}{2} b^{2} \sigma^{2} \frac{\partial^{2} C_{C}}{\partial r^{2}}+(a+b \gamma-\lambda b \sigma) \frac{\partial C_{C}}{\partial r} \\
& +\int_{-\infty}^{+\infty}\left[C_{C}(t, r+b y)-C_{C}(t, r)-b y \frac{\partial C_{C}(t, r)}{\partial r}\right] \nu(d y)-r C_{C}=0
\end{aligned}
$$


with the final condition

$$
C(r, T)=\max \left(V_{C}(r, T)-K, 0\right) .
$$

We solve for the value of the cap first and then use this value as the final data for the value of the caption.

Pricing of Floortions. Consider European Floor Call Option, option to buy this floor (a call floortion) for an amount $K$ at time $T<T_{F}$, where $T_{F}$ is an expiry time for floor with value $V_{F}(r, t), t \leq T_{F}$. Thus, this value $V_{F}$ satisfies the following PIDE (see (38)):

$$
\begin{aligned}
\frac{\partial V_{F}}{\partial t} & +\frac{1}{2} b^{2} \sigma^{2} \frac{\partial^{2} V_{F}}{\partial r^{2}}+(a+b \gamma-\lambda b \sigma) \frac{\partial V_{F}}{\partial r} \\
& +\int_{-\infty}^{+\infty}\left[V_{F}(t, r+b y)-V_{F}(t, r)-b y \frac{\partial V_{F}(t, r)}{\partial r}\right] \nu(d y) \\
& -r V_{F}+\min \left(r, r^{*}\right)=0 .
\end{aligned}
$$

with the final condition

$$
V_{C}\left(r, T_{F}\right)=1 \text {. }
$$

Then the value $C_{F}(r, t)$ of this call floor option (call floortion) satisfies the following PIDE:

$$
\begin{aligned}
\frac{\partial C_{F}}{\partial t} & +\frac{1}{2} b^{2} \sigma^{2} \frac{\partial^{2} C_{F}}{\partial r^{2}}+(a+\gamma-\lambda b \sigma) \frac{\partial C_{F}}{\partial r} \\
& +\int_{-\infty}^{+\infty}\left[C_{F}(t, r+y)-C_{F}(t, r)-y \frac{\partial C_{F}(t, r)}{\partial r}\right] \nu(d y)-r C_{F}=0 .
\end{aligned}
$$

with the final condition

$$
C_{F}(r, T)=\max \left(V_{F}(r, T)-K, 0\right) .
$$

We solve for the value of the floor first and then use this value as the final data for the value of the floortion.

Remark. One of the approach to solve these PDIEs could be numerical using different finite difference methods, see Duffy (2005).

\section{Conclusion}

We discussed how to calculate the price of zero-coupon bonds for many Gaussian and Lévy one-factor and multi-factor models of $r(t)$ using change of time method. These models include, in particular, Ornshtein-Uhlenbeck (1930), Vasicek (1977), Cox-Ingersoll-Ross (1985), continuous-time GARCH, Ho-Lee (1986), Hull-White (1990) and Heath-Jarrrow-Morton (1992) models and their various combinations. We also derive PDIE for the values of swaps, caps, floors and options on them, swaptions, captions and floortions, respectively. We apply the change of time method to price the interest rate derivatives for the interest rates $r(t)$ described by various stochastic differential equations driven by $\alpha$-stable Lévy processes. We could also apply the same techniques, i.e., change of time and PIDE, to price many interest rate derivatives for multi-factor Gaussian and Lévy interest rate models. But this discussion is outside the scope of this paper and will be considered in the future research paper, as well as the numerical solutions of PIDEs presented in the paper. 


\section{Acknowledgement.}

This research is supported by NSERC. I would like to thank the organizers of the 2008 Stochastic Modelling Symposium for their invitation to present the paper. All the remaining errors are mine.

\section{Appendix A: One-Factor and Multi-Factor Gaussian Interest Rate Models}

\subsection{One-Factor Gaussian SIRMs}

1. The Geometric Brownian Motion Model (Rendleman and BARTTER (1980)). $d r(t)=\mu r(t) d t+\sigma r(t) d W(t)$.

2. The Ornstein-Uhlenbeck (1930) Model. $d r(t)=-\mu r(t) d t+$ $\sigma d W(t)$

3. The VAsićek (1977) Model. $d r(t)=\mu(b-r(t)) d t+\sigma d W(t)$.

4. The Continuous-Time GARCH Model. $d r(t)=\mu(b-r(t)) d t+$ $\sigma r(t) d W(t)$.

5. The Cox-Ingersoll-Ross (1985) Model. $d r(t)=k(\theta-r(t)) d t+$ $\gamma \sqrt{r} d W(t)$

6. The Ho And Lee (1986) Model. $d r(t)=\theta(t) d t+\sigma d W(t)$.

7. The Hull And White (1990) Model. $d r(t)=(a(t)-b(t) r(t)) d t+$ $\sigma(t) d W(t)$

8. The Heath, Jarrow and Morton (1987) Model. Define the forward interest rate $f(t, s)$, for $t \leq s$, characterized by the following equality $P(t, u)=\exp \left[-\int_{t}^{u} f(t, s) d s\right]$ for any maturity $u . f(t, s)$ represents the instanteneous interest rate at time $s$ as 'anticipated' by the market at time $t$. It is natural to set $f(t, t)=r(t)$. The process $f(t, u)_{0 \leq t \leq u}$ satisfies an equation $f(t, u)=f(0, u)+\int_{0}^{t} a(v, u) d v+\int_{0}^{t} b(f(v, u)) d W(v)$, where the processes a and $b$ are continuous. We note, that the lasr SDE may be written in the following form: $d f(t, u)=b(f(t, u))\left(\int_{t}^{u} b(f(t, s))\right) d s+b(f(t, u)) d \hat{W}(t)$, where $\hat{W}(t)=W(t)-\int_{0}^{t} q(s) d s$ and $q(t)=\int_{t}^{u} b(f(t, s)) d s-\frac{a(t, u)}{b(f(t, u))}$.

\subsection{Multi-Factor Gaussian SIRMs}

Multi-factor models driven by Brownian motions can be obtained using various combinations of above-mentioned processes. We give one example of two-factor continuous-time GARCH SIRM:

$$
\left\{\begin{array}{l}
d r(t)=\mu(b(t)-r(t)) d t+\sigma r(t) d W^{1}(t) \\
d b(t)=\xi b(t) d t+\eta b(t) d W^{2}(t),
\end{array}\right.
$$

where $W^{1}, W^{2}$ may be correlated, $\mu, \xi \in R, \sigma, \eta>0$. 


\section{Appendix B: Solutions to the One-Factor and Multi-Factor Gaussian Interest Rate Models}

\subsection{Solution of One-Factor Gaussian SIRMs Using CTM}

We use change of time method (see Ikeda and Watanabe (1981)) to get the solutions to the following below equations (see Swishchuk (2007)). $W(t)$ below is an standard Brownian motion, and $\hat{W}$ is a $\left(\hat{T}_{t}\right)_{t \in R_{+}}$-adapted standard Brownian motion on $\left(\Omega, \mathcal{F},\left(\hat{\mathcal{F}}_{t}\right)_{t \in R_{+}}, P\right)$.

1. Geometric Brownian Motion. $d r(t)=\mu r(t) d t+\sigma r(t) d W(t)$. Solution $r(t)=e^{\mu t}\left[r(0)+\hat{W}\left(\hat{T}_{t}\right)\right]$, where $\hat{T}_{t}=\sigma^{2} \int_{0}^{t}\left[r(0)+\hat{W}\left(\hat{T}_{s}\right)\right]^{2} d s$.

2. Ornstein-Uhlenbeck Process. $d r(t)=-\mu r(t) d t+\sigma d W(t)$, Solution $r(t)=e^{-\mu t}\left[r(0)+\hat{W}\left(\hat{T}_{t}\right)\right]$, where $\hat{T}_{t}=\sigma^{2} \int_{0}^{t}\left(e^{\mu s}\left[r(0)+\hat{W}\left(\hat{T}_{s}\right)\right]\right)^{2} d s$.

3. VAsićé Process. $d r(t)=\mu(b-r(t)) d t+\sigma d W(t)$, solution $r(t)=$ $e^{-\mu t}\left[r(0)-b+\hat{W}\left(\hat{T}_{t}\right)\right]$, where $\hat{T}_{t}=\sigma^{2} \int_{0}^{t}\left(e^{\mu s}\left[r(0)-b+\hat{W}\left(\hat{T}_{s}\right)\right]+b\right)^{2} d s$.

4. Continuous-Time GARCH Process. $d r(t)=\mu(b-r(t)) d t+$ $\sigma r(t) d W(t)$. Solution $r(t)=e^{-\mu t}\left(r(0)-b+\hat{W}\left(\hat{T}_{t}\right)\right)+b$, where $\hat{T}_{t}=\sigma^{2} \int_{0}^{t}[r(0)-$ $\left.b+\hat{W}\left(\hat{T}_{s}\right)+e^{\mu s} b\right)^{2} d s$.

5. Cox-Ingersoll-Ross Process. $d r^{2}(t)=k\left(\theta-r^{2}(t)\right) d t+\gamma r(t) d W(t)$, solution $r^{2}(t)=e^{-k t}\left[r_{0}^{2}-\theta^{2}+\hat{W}\left(\hat{T}_{t}\right)\right]+\theta^{2}$, where $T_{t}=\gamma^{-2} \int_{0}^{t}\left[e^{k T_{s}}\left(r_{0}^{2}-\theta^{2}+\right.\right.$ $\left.\hat{W}(s))+\theta^{2} e^{2 k T_{s}}\right]^{-1} d s$.

6. Ho And Lee Process. $d r(t)=\theta(t) d t+\sigma d W(t)$. Solution $r(t)=$ $r(0)+\hat{W}\left(\sigma^{2} t\right)+\int_{0}^{t} \theta(s) d s$.

7. Hull And White. $d r(t)=(a(t)-b(t) r(t)) d t+\sigma(t) d W(t)$. Solution $r(t)=\exp \left[-\int_{0}^{t} b(s) d s\right]\left[r(0)-\frac{a(s)}{b(s)}+\hat{W}\left(\hat{T}_{t}\right)\right]$, where $\hat{T}_{t}=\int_{0}^{t} \sigma^{2}(s)\left[r(0)-\frac{a(s)}{b(s)}+\right.$ $\left.\hat{W}\left(\hat{T}_{s}\right)+\exp \left[\int_{0}^{s} b(u) d u\right] \frac{a(s)}{b(s)}\right] d s$.

8. Heath, Jarrow and Morton. $f(t, u)=f(0, u)+\int_{0}^{t} a(v, u) d v+$ $\int_{0}^{t} b(f(v, u)) d W(v)$. Solution $f(t, u)=f(0, u)+\hat{W}\left(\hat{T}_{t}\right)+\int_{0}^{t} a(v, u) d v$, where $\hat{T}_{t}=\int_{0}^{t} b^{\alpha}\left(f(0, u)+\hat{W}\left(\hat{T}_{s}\right)+\int_{0}^{s} a(v, u) d v\right) d s$.

\subsection{Solution of Multi-Factor Gaussian SIRMs Using CTM}

Solution of multi-factor models driven by Brownian motions can be obtained using various combinations of solutions of the above-mentioned processes, see subsection 5.1, and CTM. We give one example of two-factor ContinuousTime GARCH model driven by Brownian motions:

$$
\left\{\begin{array}{l}
d r(t)=\mu(b(t)-r(t)) d t+\sigma r(t) d W^{1}(t) \\
d b(t)=\xi b(t) d t+\eta b(t) d W^{2}(t)
\end{array}\right.
$$


where $W^{1}, W^{2}$ may be correlated, $\mu, \xi \in R, \sigma, \eta>0$.

Solution, using CTM for the first and the second equations, subsection 13.1: $r(t)=e^{-\mu t}\left[r(0)-e^{\xi t}\left(b(0)+\hat{W}^{2}\left(\hat{T}_{t}^{2}\right)\right)+\hat{W}^{1}\left(\hat{T}_{t}^{1}\right)\right]+e^{\xi t}\left[b(0)+\hat{W}^{2}\left(T_{t}^{2}\right)\right]$, where $\hat{T}^{i}$ are defined in $4 .(i=1)$ and $1 .(i=2)$, respectevely, subsection 13.1. Here, $W^{1}(t)$ and $W^{2}(t)$ are independent.

\section{References}

D. Applebaum. Levy Processes and Stochastic Calculus, Cambridge University Press, 2003.

N. Bingham and R. Kiesel. Risk-Neutral Valuations, Pricing and Hedging of Financial Derivatives, Springer Finance, London, 1998.

T. Björk, Y. Kabanov, and W. Rungaldier. Bond market structure in the presence of marked point process, Math. Finance, 1997, 7, 211-239.

T. Björk. Arbitrage Theory in Continuous Time, Oxford University Press, 1998.

F. Black, E. Derman and W. Toy. A one-factor model of interest rates and its applications to treasury bond options, Fin. Anal. J., 1990, 46(1), 33-39.

F. Black and P. Karasinski. Bond and option pricing when short rates are lognormal, Fin Anal. J., 1991, 47(4), 52-59.

A. Brace, D. Gatarek and M. Musiela. The market model of interest rate dynamics, Math. Finance, 1997, 4, 127-155.

D. Brigo and F. Mercurio. Interest Rate Models: Theory and Practice, Springer, 2001.

M. Brennan and E. Schwartz. A continuous time approximation to the pricing of bonds. Journal of of Banking and Finance, 1979, pp. 133-155.

P. Carr and D. Madan. Option valuation using the fast Fourier transform, Journal of Computational Finance, 2, 61-73, 1998.

K. Chan, A. Karoly, F. Longstaff and A. Sanders. An empirical comparison of alternative models of the short-term interest rate, J. Fin., 1992, 47, 1209-1227.

R. Cont and E. Voltchkova. Integor-differential equations for option pricing in exponential Lévy models, Finance and Stochastics, 9, 299-325, 2005.

R. Cont and E. Voltchkova. A finite difference scheme for option pricing in jump diffusion and exponential Lévy models, Internal Report CMAP, No. 513, September 2003.

G. Courtadon. The pricing of options on default-free bonds. J. of Financial and Quant. Analysis, 17 (1982), pp. 301-329.

J. Cox,J.Ingersoll and S.Ross. A theory of the term structure of interest rate. Econometrics, 53 (1985), pp. 385-407.

Q. Dai, K. Singleton. Specification analysis of affine term structure models, J. Fin., 2000, 55, 1943-1978. 
S. Das. A discrete-time approach to Poisson-Gaussian bond option pricing in the Heath-Jarrow-Morton model, J. Econ. Dynam. Control, 1999, 23, 333-369.

S. Das and S. Foresi. Exact solutions for bond and option prices with systematic jump risk, Rev. Derivatives Res., 1996, 1, 7-24.

D. Duffie. Dynamic Asset Pricing Theory, Princeton University Press, Princeton, NJ, 1992.

D. Duffie and R. Kan. A yield-factor model of interest rate, Math. Fin., 1994, 4, 379-406.

D. Duffie and R. Kan. Multi-factor term structure models, Philos. Trans. R. Soc. London, Ser. A, 1994, 347, 577-586.

D. Duffie, J. Pan and K. Singleton. Transform analysis and option pricing for affine jump-diffusions, Econometrica, 2000, 68, 1343-1376.

D. Duffy. Numerical analysis of jump diffusion models: a partial differential equation approach, Datasim, 2005.

D. Duffy. Finite Difference Methods in Financial Engineering: A Partial Differential Approach. Wiley and Sons, Chichester, 2005.

E. Eberlein and S. Raible. Term structure models driven by general Levy processes. Mathematical Finance 9, 31-53, 1999.

D. Filipovic. Consistency Problems for Heath-Jarrow-Morton Interest Rate Models. Lecture Notes in Mathematics, vol. 1760. Springer, 2001.

H. U. Gerber and E. S. W. Shiu. Option pricing by Esscher-transforms. Transactions of the Society of Actuaries, 46, 99-191, 1994.

P. Glasserman and S. Kou. The term structure of simple forward rates with jump risk, Math. Fin., 2003, 13, 383-410.

P. Glasserman and N. Merener. Numerical solution of jump diffusion LIBOR market models, Fin. Stochastics, 2003, 7, 1-27.

D. Hearth, R.Jarrow and A.Morton. Bond pricing and the term structure of the interest rates: A new mathodology. Econometrica, 60, 1 (1992), pp. $77-105$.

J. Hull and A. White. Pricing interest rate derivative securities. Review of Fin. Studies, 3,4 (1990), pp. 573-592.

T.S.Y. Ho and S.-B. Lee. Term structure movements and pricing interest rate contigent claim. J. of Finance, 41 (December 1986), pp. 1011-1029.

N. Ikeda and S. Watanabe. Stochastic Differential Equations and Diffusion Processes. North-Holland/Kodansha Ltd., Tokyo, 1981.

J. Jacod and A. Shiryaev. Limit Theorems for Stochastic Processes, Springer-Verlag, 1987; second edition (2003).

J. James and N. Webber. Interest Rate Modelling. John Wiley \& Sons, Ltd., 2002.

F. Jamshidian. An exact bond option pricing formula. J. of Finance, 44 (March 1989), pp. 205-209.

F. Jamshidian. LIBOR and swap market models and measures, Fin. Stochastics, 1, 293-330. 
D. Jara. An extension of Lévy theorem and application to financial models based on futures prices, PhD dissertation, Dept. of Math. Sciences, Carnegie Mellon University, 2000.

D. Lamperton and B.Lapeyre. Introduction to Stochastic Calculus Applied to Finance, Chapman\&Hall, 1996.

F. Longstaff and E. Schwartz. Interest rate volatility and the term structure: A two-factor general equilibrium model, J. Finance, 47(4): 1259-82.

Y. Maghsoodi. Solution of the extended CIR term structure and bond option valuation, Math. Fin., 1996, 6, 89-109.

K. Miltersen, S. Sandmann and D. Sondermann. Closed form solutions for term structure derivatives with log-normal interest rates, J. Finance, 52, 409-430.

D. Nualart and W. Schoutens. Backwards SDE and Feynman-Kac formula for Lévy processes, with application in finance, Bernoulli, 7, 761-776.

L. Ornstein and G.Uhlenbeck. On the theory of Brownian motion. Physical Review, 36 (1930), 823-841.

A. Pelsser. Efficient Methods for Valuing Interest Rate Derivatives, Springer, Berlin, 2000.

S. Raible. Levy processes in finance: theory, numerics, and empirical facts. PhD thesis, Freiburg, 2000.

R. Rebonato. Modern Pricing of Interest-Rate Derivatives: The LIBOR Market Model and Beyond, Princeton University Press, Princeton, NJ, 2000.

R. Rebonato. Interest-Rate Option Models. John Wiley \& Sons, Ltd., 1996.

R. Rendleman and B. Barttler. The pricing of options on debt securities, Journal of Financial and Quantitative Analysis, 15 (March 1980), 11-24.

J. Rosiński and W. Woyczyński. On Ito stochastic integration with respect to $p$-stable motion: inner clock, integrability of sample paths, double and multiple integrals, Ann. Probab. 14, 271-286, 1986.

K. Sato. Levy Processes and Infinitely Divisible Distributions. Cambridge Studies in Advanced Mathematics, vol. 68. Cambridge University Press, 1999.

S. Schaefer and E.Schwartz. Time-dependent and the pricing of options. J. of Finance, 42 (December 1987), pp. 1113-1128.

H. Shirakawa. Interest rate option pricing with Poisson-Gaussian forward rate curve processes, Math. Fin., 1991, 1, 77-94.

$O$. Vasicek. An equilibrium characterization of the term structure. J. of Finan. Economics, 5 (1977), pp. 177-188.

W. Schoutens. Lévy Processes in Finance. Pricing Financial Derivatives. Wiley \& Sons, 2003.

A. Swishchuk. Modeling of Variance and Volatility Swaps for Fiancial Markets with Stochastic Volatility, WILMOTT Magazine, 2004, September Issue, Technical Article No 2, pp. 64-72.

A. Swishchuk. Change of time method in mathematical finance, Canad. Appl. Math. Quart., vol. 15, No. 3, 2007, 32p. 
P. Wilmott, J. Dewynne and S. Howison. The Mathematics of Financial Derivatives. A Student Introduction, Cambridge University Press, 1995. 